\title{
Genome-wide chemical mutagenesis screens allow unbiased saturation of the cancer genome and identification of drug resistance mutations
}

\author{
Jonathan S. Brammeld, ${ }^{1}$ Mia Petljak, ${ }^{1}$ Inigo Martincorena, ${ }^{1}$ Steven P. Williams, ${ }^{1}$ \\ Luz Garcia Alonso, ${ }^{2}$ Alba Dalmases, ${ }^{3}$ Beatriz Bellosillo, ${ }^{3}$ Carla Daniela Robles-Espinoza, ${ }^{4}$ \\ Stacey Price, ${ }^{1}$ Syd Barthorpe, ${ }^{1}$ Patrick Tarpey, ${ }_{1}^{1}$ Constantine Alifrangis, ${ }^{1}$ \\ Graham Bignell, ${ }^{1}$ Joana Vidal, ${ }^{5}$ Jamie Young, ${ }^{1}$ Lucy Stebbings, ${ }^{1}$ Kathryn Beal, ${ }^{1}$ \\ Michael R. Stratton, ${ }^{1}$ Julio Saez-Rodriguez, ${ }^{2,6}$ Mathew Garnett, ${ }^{1}$ Clara Montagut, ${ }^{5}$ \\ Francesco lorio, ${ }^{2,7}$ and Ultan McDermott ${ }^{1,7}$ \\ ${ }^{1}$ Wellcome Trust Sanger Institute, Hinxton CB10 1SA, United Kingdom; ${ }^{2}$ European Molecular Biology Laboratory - European \\ Bioinformatics Institute, Cambridge CB10 1SA, United Kingdom; ${ }^{3}$ Pathology Department, Hospital del Mar, 08003 Barcelona, Spain; \\ ${ }^{4}$ Laboratorio Internacional de Investigación sobre el Genoma Humano, Universidad Nacional Autónoma de México, Campus \\ Juriquilla, Santiago de Querétaro 76230, Mexico; ${ }^{5}$ Cancer Research Program, FIMIM and Medical Oncology Department, Hospital \\ del Mar, 08003 Barcelona, Spain; ${ }^{6}$ RWTH Aachen University Hospital, 52062 Aachen, Germany
}

\begin{abstract}
Drug resistance is an almost inevitable consequence of cancer therapy and ultimately proves fatal for the majority of patients. In many cases, this is the consequence of specific gene mutations that have the potential to be targeted to resensitize the tumor. The ability to uniformly saturate the genome with point mutations without chromosome or nucleotide sequence context bias would open the door to identify all putative drug resistance mutations in cancer models. Here, we describe such a method for elucidating drug resistance mechanisms using genome-wide chemical mutagenesis allied to next-generation sequencing. We show that chemically mutagenizing the genome of cancer cells dramatically increases the number of drug-resistant clones and allows the detection of both known and novel drug resistance mutations. We used an efficient computational process that allows for the rapid identification of involved pathways and druggable targets. Such a priori knowledge would greatly empower serial monitoring strategies for drug resistance in the clinic as well as the development of trials for drug-resistant patients.
\end{abstract}

[Supplemental material is available for this article.]

Despite an increasing array of new cancer therapies, drug resistance is an almost universal phenomenon that is likely due to the presence of rare subclonal populations that act as a reservoir for resistance mutations. The emergence of drug resistance ultimately proves fatal for the majority of patients, and therefore the early detection of resistance and the identification of novel resensitization strategies is a subject of intense activity.

Previously, the identification of drug resistance genes has relied on either rebiopsy of cancer patients following the development of resistance or the use of cancer cell lines made resistant by exposure in vitro to drug over many weeks. Both approaches can suffer from inherent biases. With respect to the former, biopsy of a single resistant site of disease may miss alternate resistance mechanisms in other metastatic sites (Van Allen et al. 2014). Equally, serial drug exposure in cancer cell lines will favor preexisting drug-resistant clones that are specific for that cell line and may not represent the entire spectrum of resistance mechanisms for that treatment.
For these reasons, there is considerable interest in the use of forward genetic screens capable of engineering into the cancer genome mutational events that can be tested for their ability to cause drug resistance in an unbiased fashion. Such screens, if sufficiently unbiased, could in theory capture the entire breadth of genetic resistance mechanisms for any drug. Recent studies have demonstrated the power of both genome-wide gain- and loss-offunction screens using CRISPR/Cas9, lentiviral shRNA, and largescale open-reading frame technologies to identify clinically relevant drug resistance mechanisms in cancer (Hu and Zhang 2016). However, these screens all fail to capture a third important mechanism of drug resistance, namely that of point mutations. Point mutations account for resistance in large numbers of patients receiving targeted therapies in melanoma, colon and lung cancers, and chronic myeloid leukemia (Supplemental Table S1; Kobayashi et al. 2005; Katayama et al. 2012; Montagut et al. 2012; Ohashi et al. 2012; Bettegowda 2014; Long et al. 2014; Van Allen et al. 2014; Wagle et al. 2014; Arena et al. 2015; Russo et al. 2015; Siravegna et al. 2015; Thress et al. 2015).

(c) 2017 Brammeld et al. This article, published in Genome Research, is available under a Creative Commons License (Attribution 4.0 International), as described

at http://creativecommons.org/licenses/by/4.0/.

\footnotetext{
${ }^{7}$ Co-senior authors

Corresponding authors: um1@sanger.ac.uk, iorio@ebi.ac.uk Article published online before print. Article, supplemental material, and publication date are at http://www.genome.org/cgi/doi/10.1101/gr.213546.116. Freely available online through the Genome Research Open Access option.
} 
$\mathrm{N}$-Ethyl- $\mathrm{N}$-nitrosourea (ENU) has been used as a potent mutagen in mouse models of development for over four decades (Acevedo-Arozena et al. 2008). Exposure results in the efficient generation of random point mutations throughout the cell genome (Tokunaga et al. 2014). We therefore tested whether, in cancer cell line models, ENU could be used to mutagenize the genome and enable expansion of drug-resistant cells following the application of a targeted agent. As proof of concept, we chose to investigate whether this approach could identify all clinically demonstrated resistance mutations in colorectal cancer patients treated with the EGFR monoclonal antibody Cetuximab (Van Cutsem et al. 2009). In the clinic, resistance in such patients is heavily driven by point mutations, and a decade of clinical studies has identified the vast majority of the resistance mutations. This "ground truth" should in theory allow us to define how well a saturation mutagenesis screen can identify clinically relevant resistance mutations.

We used a sequencing and informatics approach to detect novel resistance mutations from next-generation sequence data and to detect statistical enrichment for mutually exclusive mutations in specific signaling pathways comprising more than 8000 genes at the sample population level. Our mutagenesis screen was able to successfully identify all known drug resistance mutations to Cetuximab previously observed in the clinic as well as a novel mutation that we subsequently identified in a colorectal cancer patient. We suggest that this approach is a powerful and facile means to draw the landscape of point mutations that confer resistance to targeted therapies. Such knowledge could be used to discover therapeutic strategies to resensitize resistant tumors as well as identify which genes should be prioritized for noninvasive monitoring during treatment using plasma DNA sequencing.

\section{Results}

\section{ENU exposure confers stable resistance to Cetuximab in colon cancer cells}

We screened 51 colorectal cancer cell lines with a concentration range of the EGFR monoclonal antibody Cetuximab and assessed viability after $6 \mathrm{~d}$ (Fig. 1A). In keeping with clinical experience of the genetic factors that underpin response to this drug, those cell lines wild-type for $K R A S / N R A S / B R A F$ (green bars) exhibited heightened sensitivity to Cetuximab (Douillard et al. 2013). We therefore chose two of these lines, CCK-81 and NCI-H508, to use in the ENU resistance experiment. Both cell lines additionally demonstrated Cetuximab sensitivity in long-term clonogenic survival assays (Supplemental Fig. S1). Moreover, CCK-81 has features of microsatellite instability (MSI), whereas NCI-H508 is microsatellite stable (MSS). MSI is detected in $16 \%$ of colorectal cancers and is associated with a different phenotype and clinical outcome compared to MSS cancers. The CCK-81 cell line was exposed to a dose range of ENU $(0.01-1 \mathrm{mg} / \mathrm{mL})$ for $24 \mathrm{~h}$, following which the mutagenized cells were treated with Cetuximab $(10 \mu \mathrm{g} / \mathrm{mL})$ for 8 consecutive weeks. The number of drug-resistant colonies was counted at the end of the experiment. Importantly, we observed no drug-resistant colonies in the absence of ENU (Fig. 1B). With increasing ENU concentration, we observed a linear increase in both the number of drug-resistant colonies (left $y$-axis, blue bars) as well as the number of mutations per clone (right $y$ axis, green triangles). We subsequently used a concentration of ENU $(0.1 \mathrm{mg} / \mathrm{mL})$ that resulted in minimal viability effect in both cell lines (Methods). We next treated NCI-H508 cells with ENU (0.1 $\mathrm{mg} / \mathrm{mL}$ ) for $24 \mathrm{~h}$ followed by weekly Cetuximab treatment for eight weeks. Drug-resistant colonies were picked, expanded in culture, and 72 were submitted for whole-exome Illumina sequencing (a total of 14 CCK-81 and 58 NCI-H508 colonies). Data were analyzed for substitutions and insertions/deletions to enable an estimation of the number of ENU-associated mutations per $\mathrm{Mb}$ of exome and to detect novel (and putative drug resistance) mutations (Supplemental Table S2). We then performed clonogenic survival assays on a subset of resistant clones and confirmed robust and stable resistance to Cetuximab (Fig. 1C).

\section{The spectrum of ENU-induced mutations}

The ability of any mutagenesis screen to capture a particular phenotype is strongly dependent on its ability to evenly saturate the genome with all six possible classes of base substitution type (expressed as the pyrimidine of a mutated Watson-Crick base pair, $\mathrm{C}>\mathrm{A}, \mathrm{C}>\mathrm{G}, \mathrm{C}>\mathrm{T}, \mathrm{T}>\mathrm{A}, \mathrm{T}>\mathrm{C}, \mathrm{T}>\mathrm{G}$ ). On average, we detected 470 novel mutations per exome in each clone (mean 570 and 446 in CCK-81 and NCI-H508 clones, respectively), for a total of 33,857 (Supplemental Table S2). The mutations were almost exclusively composed of base substitutions ( $96 \%$ of total). A third of such mutations were nonsynonymous (missense) variants within the coding exon of a gene, where resistance mutations are more likely to occur (Fig. 2A). Only 4\% were potential loss-of-function truncating mutations (frameshift indels or nonsense mutations). The remaining mutations were predominantly either silent or intronic. Significantly, analysis of exome sequence data across all 72 clones (regardless of whether MSS or MSI) revealed that of the six possible classes of base substitution, only $\mathrm{C}>\mathrm{G}$ substitutions are less well represented (3\% of all substitution base changes) (Fig. 2A). The mutation spectrum in ENU-derived clones was similar regardless of whether the cells came from a MSI or MSS background (Supplemental Fig. S2). There was no evidence of a significant bias toward mutations in coding genes in any particular chromosome or indeed any specific region within a chromosome (Fig. 2B; Supplemental Fig. S3). However, given these 33,857 mutations are an admixture of those caused by ENU, background mutational processes, and private subclonal variants, we elected to use a mathematical approach to specifically extract the ENU signature from the data and more accurately determine the mutation spectrum of ENU mutations.

The non-negative matrix factorization algorithm has previously been used to detect the presence of mutational signatures in human cancers, including from defects in DNA mismatch repair and altered activity of the error-prone polymerase POLE (http:// cancer.sanger.ac.uk/cosmic/signatures) (Alexandrov et al. 2013b). It extracts signatures based on a 96-mutation classification that incorporates the six base substitution types described above as well as the immediate flanking sequence context of the mutated base (four possible $5^{\prime}$ and four possible $3^{\prime}$ bases). In our data, it revealed a distinct and unique signature that was represented across almost all trinucleotide contexts in both CCK-81 and NCI-H508 clones and not previously detected in prior tumor studies, including a panel of 51 colorectal cancer cell lines (data not shown) (Fig. 2C; Supplemental Fig. S4). This signature ("Signature A") is likely one of ENU exposure. Reassuringly, the pattern of base substitutions that comprise this signature was almost identical to that seen across the entire set of substitutions detected in the ENU-derived clones, with again only $\mathrm{C}>\mathrm{G}$ substitutions seen at lower frequency (Fig. 2C). Thus, using this approach it should be feasible to generate the majority of theoretical coding point mutations for drug resistance across the entire genome.

\section{Genome Research}

www.genome.org 
A
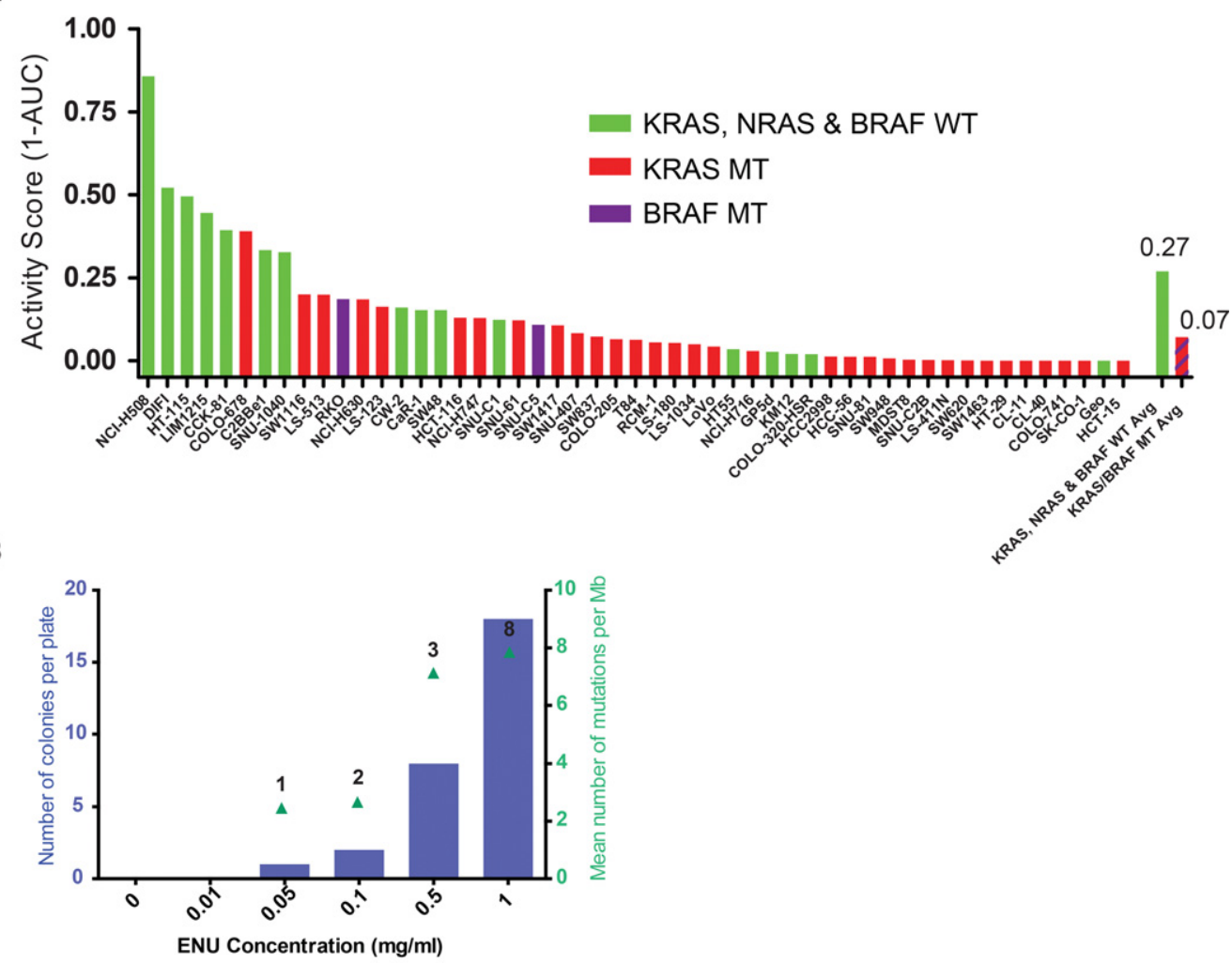

C
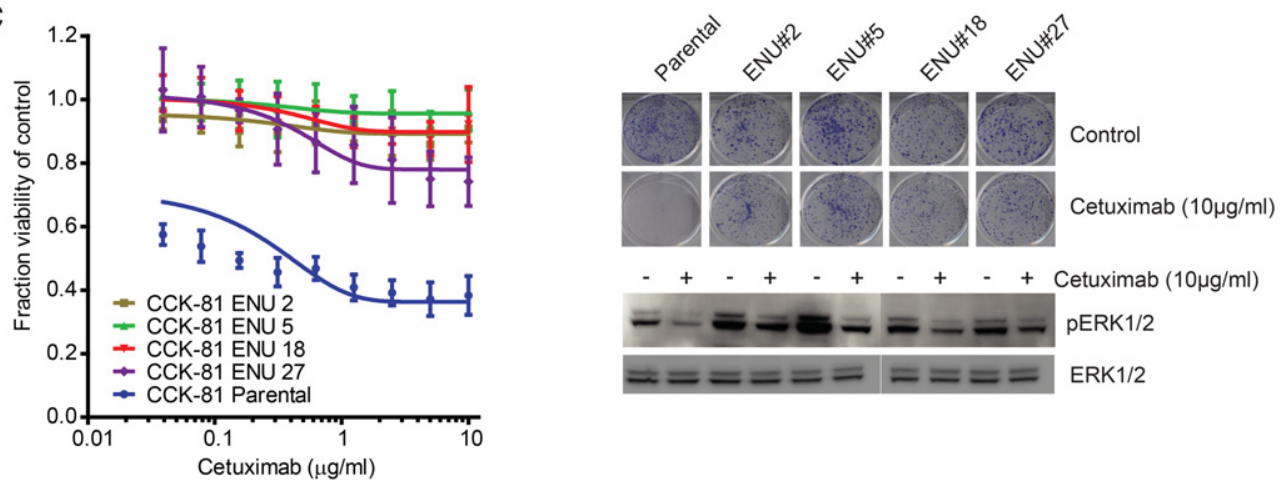

Figure 1. Genome-wide chemical mutagenesis screens to define pathways of drug resistance in cancer. $(A)$ Cetuximab viability screen in colorectal cancer cell lines. Fifty-one colorectal cancer cell lines were screened with a concentration range of the EGFR monoclonal antibody Cetuximab and viability (as measured by the Activity Score, 1-AUC) was measured after $6 \mathrm{~d}$. The KRAS, NRAS, and BRAF mutation status of each line is indicated. The mean Activity Score for those cell lines wild-type for all three genes (green bar) versus those with a canonical driver mutation in at least one gene (red bar) is indicated in the last two columns. (B) Dose-dependent effect of ENU on mutation burden and drug resistance. CCK-81 colorectal cancer cells were treated for $24 \mathrm{~h}$ with increasing concentrations of ENU ( $x$-axis, $0.01-1 \mathrm{mg} / \mathrm{mL}$ ) and then weekly with Cetuximab $(10 \mu \mathrm{g} / \mathrm{mL}$ ) for 8 wk to allow drug-resistant colonies to develop. The number of resistant colonies per plate were counted for each ENU concentration (blue bars) and submitted for whole-exome sequencing to calculate the mean number of mutations per $\mathrm{Mb}$ (green triangles). Numbers above each triangle indicate the number of clones sequenced at that concentration. (C) ENU mutagenesis generates stably drug-resistant clones. A subset of Cetuximab-resistant clones generated in the CCK-81 cell line following exposure to ENU and subsequent serial weekly Cetuximab treatment were picked from the plate and expanded separately out of drug for $4 \mathrm{wk}$. Twenty-one day clonogenic survival assays in four clones (along with the parental CCK-81 cell line) treated with Cetuximab $10 \mu \mathrm{g} / \mathrm{mL}$ confirmed that resistance to the EGFR monoclonal antibody had been maintained and was stable. A 6-d viability assay of cells treated with a concentration range of Cetuximab (right) demonstrates resistance of ENU clones at all concentrations. Immunoblot analysis of effect of Cetuximab treatment $(10 \mu \mathrm{g} / \mathrm{mL})$ for $6 \mathrm{~h}$ confirms persistence of MAPK signaling in ENU clones.

As expected, we detected a signature of MSI ("Signature B") in the combined mutational catalog for the CCK-81 clones but surprisingly also in the NCI-H508 clones (Supplemental Fig. S4; Alexandrov et al. 2013a). On closer examination, this signature was the result of two hypermutator clones in the NCI-H508 muta- tional catalog (red arrows) (clones NCI-H508_26 and NCIH508_40) (Supplemental Fig. S5). These clones had mutation rates as high as any of the MSI CCK-81 clones and increased numbers of small insertions and deletions. This would be in keeping with a defect in the mismatch repair pathway (Supplemental Table S2). 
A

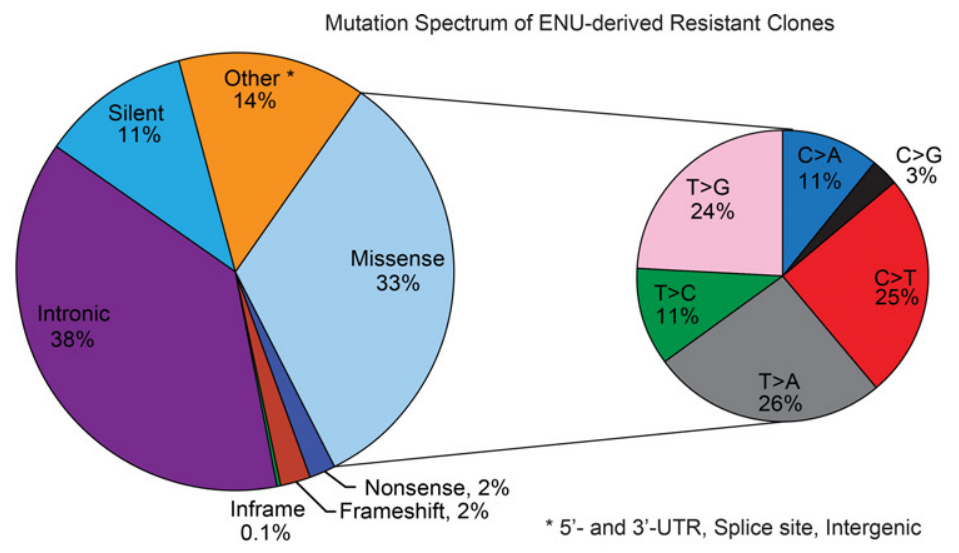

B

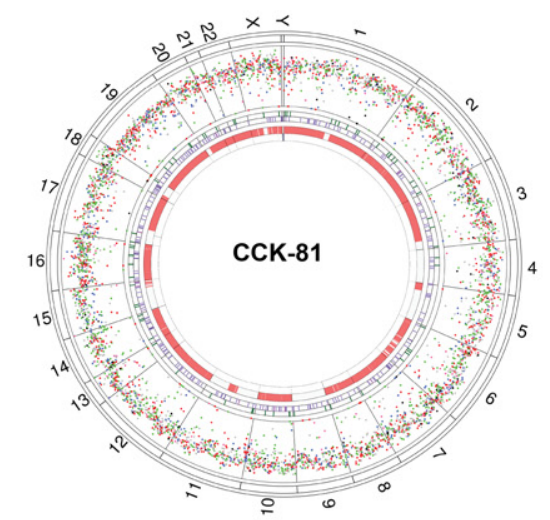

Substitutions

- $\mathrm{C}>\mathrm{A} \bullet \mathrm{T}>\mathrm{A}$

- $C>G$ - $T>C$

- $\mathrm{C}>\mathrm{T} \bullet \mathrm{T}>\mathrm{G}$

Copy Number

- Deletion

- Low level amplification

- High level amplification

Insertions \& Deletions

I. Complex

- Insertion

- Deletion

- Deletion m-homology

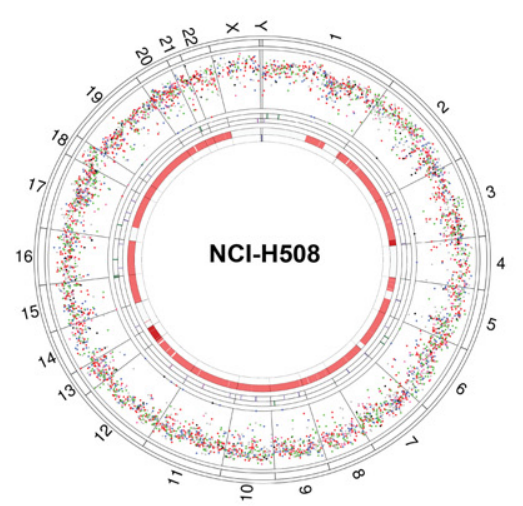

C
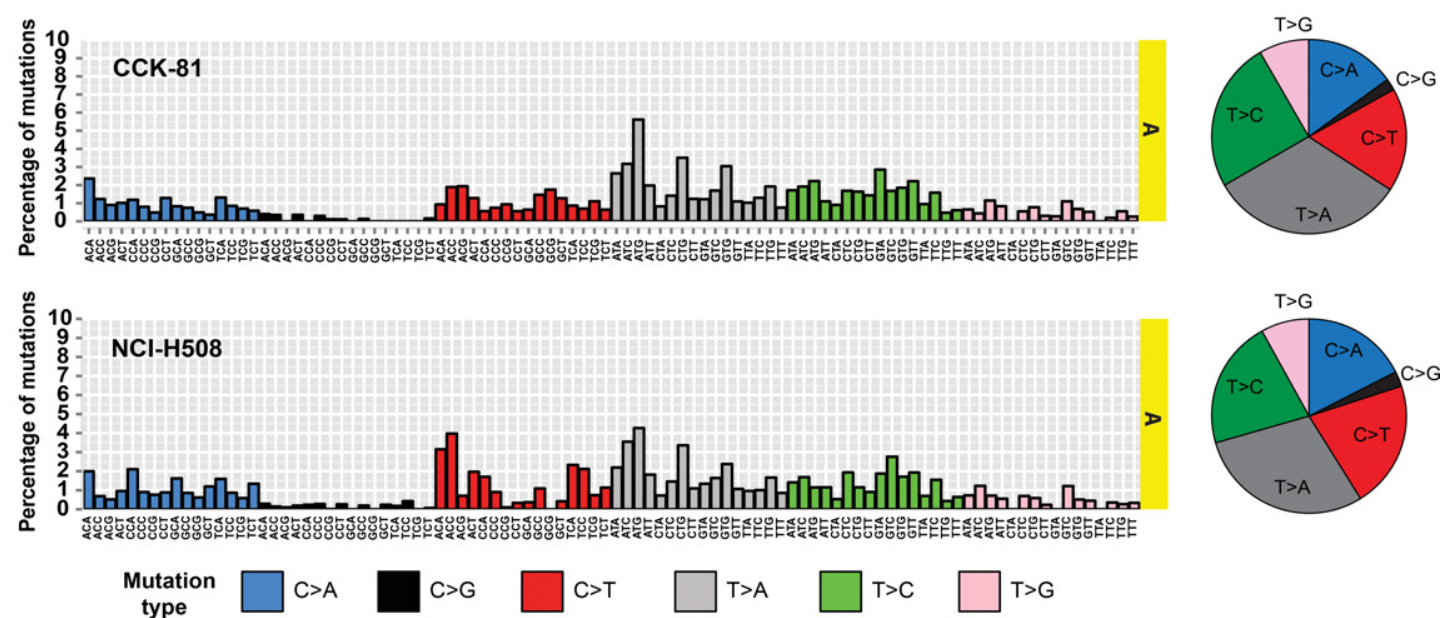

Figure 2. The spectrum of ENU-induced mutations. (A) Spectrum of mutations in ENU-derived drug-resistant clones. A pie chart representation of the proportion of 33,857 mutations detected in CCK-81 and $\mathrm{NCl}-\mathrm{H} 508$ clones categorized according to mutation type. For the missense mutations, there is a further representation of the proportion of mutations falling within the six possible nucleotide base substitutions $(C>A, C>C, C>T, T>A, T>C, T>C)$. $(B)$ Circos plots of mutations in CCK-81 and NCl-H508 clones. All substitution mutations in CCK-81 or NCl-H508 clones are represented by their intermutation distance per chromosome. Each chromosome is composed of all coding exons in genes found within that chromosome. The four circles in each plot, from outermost inward, represent the human chromosomes, substitution mutations, insertion mutations, deletion mutations, copy number gains, and copy number losses. Low- and high-level amplification refers to 2-4 and 4-8 copies, respectively. (C) The mutational signature of ENU. The trinucleotide representation of ENU mutations in CCK-81 and NCl-H508 drug-resistant colonies is displayed as the distribution of mutations for all 96 possible combinations of mutations generated as a result of ENU exposure. Adjacent pie charts display the relative distribution of each of the six classes of possible base substitutions for the CCK-81 and $\mathrm{NCl}-\mathrm{H} 508$ clones.

Clone NCI-H508_26 was found to harbor a novel nonsense (stopgained) mutation in the mismatch repair gene MLH1, and clone NCI-H508_40 harbored a nonsense mutation in the DNA repair gene EXO1. Two other clones (black arrows) also have elevated mutation rates that may be the result of gaining nonsense mutations in $P O L Q$ a gene involved in DNA damage repair. These gave rise to

\section{Genome Research}

www.genome.org 
the third signature of unknown origin detected in NCI-H508 clones ("Signature C") (Supplemental Fig. S4).

\section{ENU mutagenesis identifies clinically relevant resistance mutations and pathways}

A challenge in the identification of drug resistance mutations in ENU-derived clones is that each clone harbors many hundreds of "passenger" mutations in addition to that conferring resistance. We hypothesized that with a sufficient population of individual resistant clones it might become feasible to use statistical enrichment for nonsynonymous coding mutations in specific pathways to help identify drug resistance mutations. We therefore used a statistical framework (SLAPenrich) to identify whether genetic alterations observed in multiple samples are enriched within a specific pathway in a statistically significant manner using a network of 8056 unique genes (https://github.com/saezlab/SLAPenrich) (Iorio et al. 2016). Once significantly enriched pathways are identified, SLAPenrich applies a final filter based on the tendency for genes in a positively selected pathway to be mutated in a mutually exclusive manner. When applying this method to the set of ENUmutations across the 72 Cetuximab-resistant clones, we found several statistically enriched pathways (false discovery rate [FDR] $<5 \%$ ) (Supplemental Table S3). The pathway most significantly enriched with mutations, "Signaling to P38 via RIT and RIN," contains many of the key genes of the canonical MAP kinase pathway (Fig. 3A). In total, we were able to identify credible resistance mutations in 42 of the 72 resistant clones (59\%) (Supplemental Table S4). We detected credible resistance mutations in all of the genes previously found clinically to confer resistance to EGFR therapy in colorectal cancer (Supplemental Table S1). EGFR, KRAS, NRAS, $B R A F$, and MAP2K1 (also known as MEK1) were each found to be mutated in three or more clones and in a mutually exclusive manner. There was no clear difference in the frequency of specific mutations between NCI-H508 and CCK-81. Furthermore, 38/42 (90\%) of these putative resistance mutations have previously been identified in colorectal patients developing resistance to Cetuximab. The most frequently observed ENU resistance mutation was that of BRAF p.V600E (13/42 clones), followed by NRAS p.Q61K (8/42) and KRAS p.G12C (4/42) (Fig. 3B,C; Supplemental Fig. S6). These mutations are all canonical driver mutations in tumorigenesis and known to activate oncogenic signaling and confer resistance to Cetuximab both experimentally and clinically (Diaz et al. 2012; Misale et al. 2012). We also detected EGFR mutations in three of the resistant clones. The EGFR I491K substitution has been shown to induce structural changes to the extracellular domain of EGFR such as to prevent Cetuximab binding and confer resistance (Montagut et al. 2012). Of note, additional mutations also present in the "Signaling to P38 via RIT and RIN" network (e.g., JAK1, IL6R, RAF1) were not taken forward for investigation as these mutations were present in clones also harboring other more credible drivers of drug resistance and were present at low frequency (fewer than three clones) (Supplemental Fig. S7).

\section{A mutation enrichment analysis identifies drug resistance genes}

Recent studies of large mutational data sets from cancer sequencing studies have used statistical approaches that consider the mutation spectrum, the sequence of each gene, the impact of coding substitutions (synonymous, missense, nonsense, splice site), and the variation of the mutation rate to detect novel cancer genes (Nik-Zainal et al. 2016). We adapted this " $d_{\mathrm{N}} / d_{\mathrm{S}}$ " (nonsynonymous/synonymous) method to analyze the mutations identified in the ENU drug-resistant clones (Methods). As this approach was designed for the analysis of unrelated tumor samples and we have instances of clones sharing mutations that reflect a common subclonal origin within the original parental cell lines, we first condensed the $72 \mathrm{ENU}$ clones into 19 representative groups, each the union of all the mutations in clones sharing more than three mutations. Three genes were identified as having a pattern of mutations supporting being under positive selection, namely NRAS, KRAS, and MAP2K1 (FDR <0.05) (Supplemental Table S5). After these, the next highest ranked gene was $B R A F$; although it was of borderline significance $(\mathrm{FDR}=0.0508)$, it is a very strong candidate for a resistance gene because $4 / 6$ of the mutations are at the canonical p.V600 locus.

\section{ENU mutagenesis identifies novel resistance mutations in MAP2KI}

Recently, two studies of plasma DNA sequencing in colorectal cancer patients undergoing treatment with EGFR monoclonal antibodies jointly identified the first MAP2K1 codon K57 resistance mutations (p.K57T and p.K57N) (Russo et al. 2015; Siravegna et al. 2015). In our study, we also identified MAP2K1 mutations at the K57 codon (p.K57N, p.K57E) as well as at two sites not previously reported (p.F53L, p.C121S) (Supplemental Table S4). We therefore sequenced these MAP2K1 loci (together with additional mutation hotspots in 34 other genes) in a series of plasma DNA samples collected from 22 colorectal cancer patients who acquired resistance to treatment with EGFR therapies (either Cetuximab or Panitumumab) after an initial response. In addition to all of the known canonical resistance mutations (in KRAS, NRAS, and $B R A F$ ), we detected in one such patient a novel p.F53L $M A P 2 K 1$ mutation predicted by our screen to be a resistance mutation (Table 1; Fig. 4A). As previously reported, we detected more than one likely resistance mutation in a number of these patients, in keeping with different metastatic sites evolving different resistance mechanisms.

To functionally validate the resistance effects of these MAP2K1 mutations, we treated CCK-81 cells expressing the novel p.F53L and p.C121S mutations as well as the previously identified p.K57N mutation with Cetuximab (alongside empty vector and wild-type MAP2K1 controls). We found that all of our candidate resistance mutations induced resistance to Cetuximab, and the strength of the resistance effect for the mutations was comparable to that conferred by overexpression of the MET receptor tyrosine kinase, a previously identified resistance mechanism (Fig. 4B, left; Bardelli et al. 2013). Long-term growth inhibition assays similarly showed robust and durable resistance to Cetuximab in the MAP2K1 mutant cells (Fig. 4B, right). Immunoblot analysis demonstrated elevated constitutive phosphorylation of ERK1/2 as well as a failure to completely suppress pERK $1 / 2$ expression following Cetuximab treatment in all of the MAP2K1 mutant clones (Fig. 4C).

\section{Rational targeting of pathways can resensitize drug-resistant mutants to Cetuximab}

Constitutive EGFR signaling in solid tumors activates a number of downstream pro-survival/proliferation pathways including PKC, PI3K/AKT/mTOR, JAK-STAT, and MAPK (Supplemental Fig. S8A; Shostak and Chariot 2015). In EGFR-dependent cells, treatment with EGFR inhibitors affects cell survival by shutting down such processes. Identification of the key signaling pathways that underpin drug resistance opens up the possibility of rationally targeting key components of such resistance pathways and thus resensitizing cells. The creation of mutagenized resistant cell lines, either through 
A

SLAPenrich FDR $1.59 \mathrm{e}-11 \%$

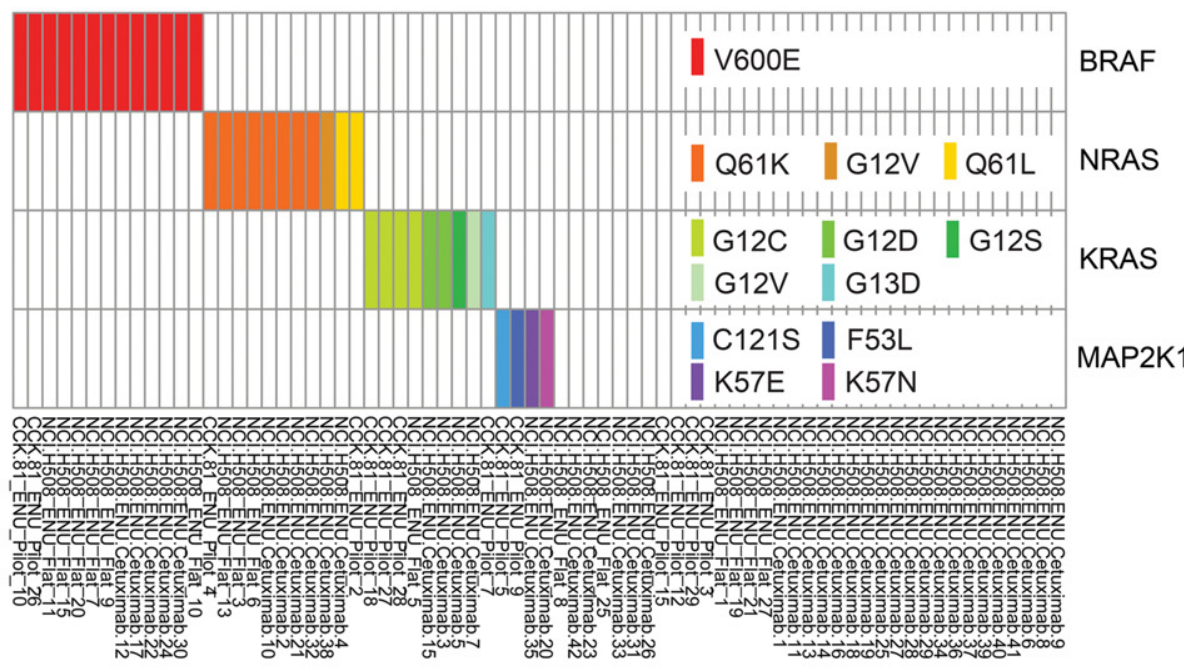

B

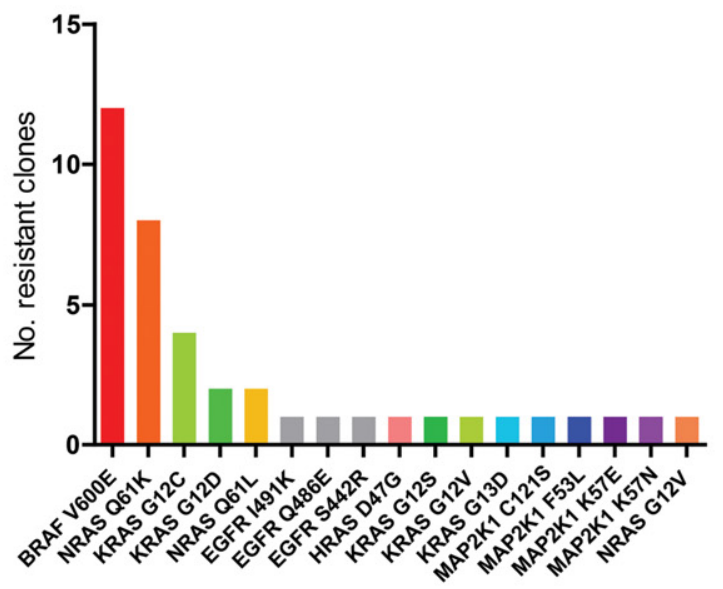

C

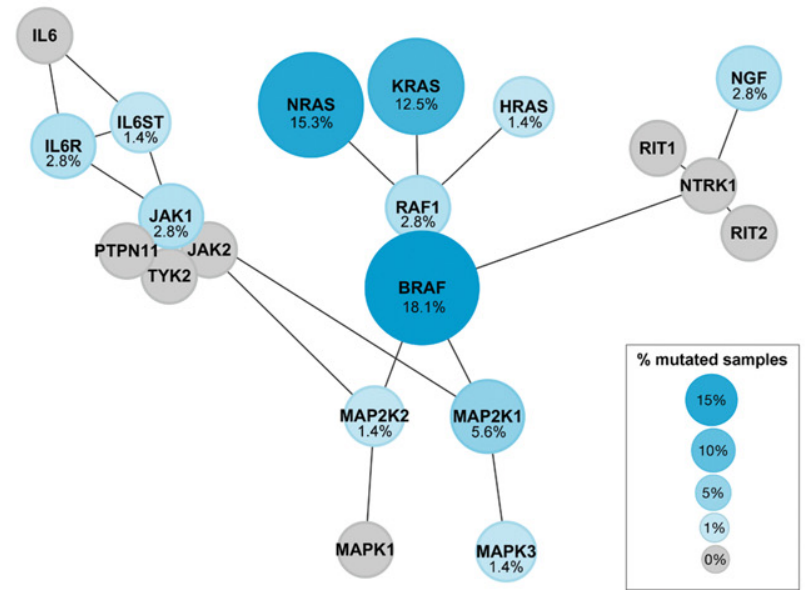

Figure 3. ENU mutagenesis identifies clinically relevant mutations and pathways. (A) The pathway most significantly enriched with mutations, "Signaling to P38 via RIT and RIN," contains many of the key genes of the canonical MAP kinase pathway, and those are mutated in a mutually exclusive manner. Here, we show those genes mutated in at least three individual clones. Whole-exome sequence from 72 Cetuximab resistant ENU clones was used to identify pathways using the SLAPenrich algorithm. Amino acid substitutions are labeled for the subset of genes most frequently mutated in the pathway and/or demonstrating mutual exclusivity with other mutations. (B) Cetuximab resistance mutations. Frequency of likely ENU-derived drug resistance mutations across 42 resistant clones. The amino acid consequence of each mutation is indicated with the gene name. (C) A visual representation of the all mutated genes that comprise the "Signaling to P38 via RIT and RIN" pathway, indicating gene-gene interactions and the hierarchy of signaling. Only those genes mutated in at least three clones were taken forward for validation.

\section{Genome Research}

www.genome.org 
Table 1. Identification of genetic alterations associated with resistance to anti-EGFR antibodies in plasma samples from 22 colorectal cancer patients

\begin{tabular}{|c|c|c|c|c|c|c|}
\hline Patient & Therapy & Resistance & Resistance mutation & $\begin{array}{c}\text { Mutant allele } \\
(\%)\end{array}$ & $\begin{array}{l}\text { Oncogenic } \\
\text { alteration in } \\
\text { COSMIC }\end{array}$ & Druggable \\
\hline 1 & FOLFIRI + Cetuximab & Acquired & KRAS p.Q61H & $47 \%$ & YES & NO \\
\hline 2 & FOLFIRI + Cetuximab & Acquired & ERBB2 amplification & - & YES & YES \\
\hline \multirow{2}{*}{3} & \multirow{2}{*}{ FOLFIRI + Cetuximab } & \multirow{2}{*}{ Acquired } & KRAS p.G12V & $36 \%$ & YES & NO \\
\hline & & & MAP2K1 p.F53L & $14 \%$ & YES & YES \\
\hline 4 & Irinotecan+Cetuximab & Acquired & ND & - & - & - \\
\hline 5 & Irinotecan+Cetuximab & Acquired & KRAS p.Q61H & $61 \%$ & YES & NO \\
\hline 6 & Irinotecan+Cetuximab & Acquired & ND & - & - & - \\
\hline 7 & FOLFOX + Cetuximab & Acquired & EGFR p.S492R & $1 \%$ & - & YES \\
\hline 8 & FOLFOX + Cetuximab & Acquired & KRAS p.G12S & $14 \%$ & YES & NO \\
\hline \multirow{2}{*}{9} & \multirow{2}{*}{ Irinotecan + Cetuximab } & \multirow{2}{*}{ Acquired } & KRAS p.G12A & $6 \%$ & YES & NO \\
\hline & & & NRAS p.Q61H & $11 \%$ & YES & NO \\
\hline 10 & Irinotecan + Cetuximab & Acquired & KRAS p.A146T & $26 \%$ & YES & NO \\
\hline \multirow{2}{*}{11} & \multirow{2}{*}{ FOLFOX+ Cetuximab } & \multirow{2}{*}{ Acquired } & NRAS p.Q61L & $31 \%$ & YES & NO \\
\hline & & & EGFR p.S492R & $6 \%$ & - & YES \\
\hline 12 & Irinotecan + Cetuximab & Acquired & ND & - & - & - \\
\hline 13 & Irinotecan + Cetuximab & Acquired & BRAF p.V600E & $14 \%$ & YES & YES \\
\hline 14 & Irinotecan + Cetuximab & Acquired & NRAS p.Q61K & $35 \%$ & YES & NO \\
\hline 15 & Irinotecan + Cetuximab & Acquired & EGFR p.R451C & $6 \%$ & - & - \\
\hline 16 & Irinotecan + Cetuximab & Acquired & ND & $13 \%$ & YES & YES \\
\hline 17 & Panitumumab & Acquired & ND & - & - & - \\
\hline 18 & Irinotecan + Cetuximab & Acquired & EGFR p.S464L & - & - & YES \\
\hline 19 & FOLFIRI + Cetuximab & Acquired & KRAS amplification & - & YES & - \\
\hline 20 & FOLFIRI + Cetuximab & Acquired & ND & - & - & - \\
\hline 21 & Cetuximab & Acquired & BRAF p.V600E & $6 \%$ & YES & YES \\
\hline 22 & Irinotecan+Cetuximab & Acquired & EGFR p.K467T & $15 \%$ & - & - \\
\hline
\end{tabular}

(FOLFIRI) folinic acid, 5-fluorouracil and irinotecan; (FOLFOX) folinic acid, 5-fluorouracil and oxaliplatin; (ND) no resistance mutation detected. The table lists putative genetic mechanisms of acquired resistance to anti-EGFR therapies that were identified in ctDNA of 22 patients. The novel MAP2K1 mutation is indicated in red.

the ENU screen or through the deliberate genetic modification of the parental cell line for specific mutations, allowed us the opportunity for such experiments to be carried out in vitro. As in clinical practice, the pathway most frequently mutated in the drug-resistant CCK-81 and NCI-H508 ENU clones converges toward MAPK family members, and targeting these nodes might be expected to overcome resistance (Fig. 3C). For example, a Cetuximab-resistant CCK-81 BRAF V600E mutant clone (ENU-10) was resensitized when the EGFR monoclonal antibody was combined with the BRAF inhibitor Dabrafenib (Supplemental Fig. S9A). In the mutant cells, the activating $B R A F$ mutation would enable such cells to continue to signal through the MAPK pathway (and survive) despite EGFR blockade (Supplemental Fig. S8B). Thus, only by targeting both EGFR and BRAF in combination can all the relevant survival effectors be silenced and the viability of the cells reduced.
Cetuximab-resistant clones harboring mutations (in KRAS, NRAS, and MAP2K1) that would be predicted to activate MAPK signaling were resensitized when a MEK inhibitor (Trametinib) was combined with Cetuximab (Supplemental Fig. S9B). Similarly, combining Cetuximab with Trametinib almost completely resensitized the resistant MAP2K1 mutant CCK-81 cells (Fig. 5A,B). Indeed, such a combination has already been suggested as putative therapeutic strategy for colon cancer patients (Misale et al. 2014).

\section{Discussion}

We proposed at the onset of this study that regardless of what sequencing technology is used to detect resistance point mutations, some a priori knowledge of the likely drug resistance candidates should greatly increase the sensitivity of such assays. Identifying 
A
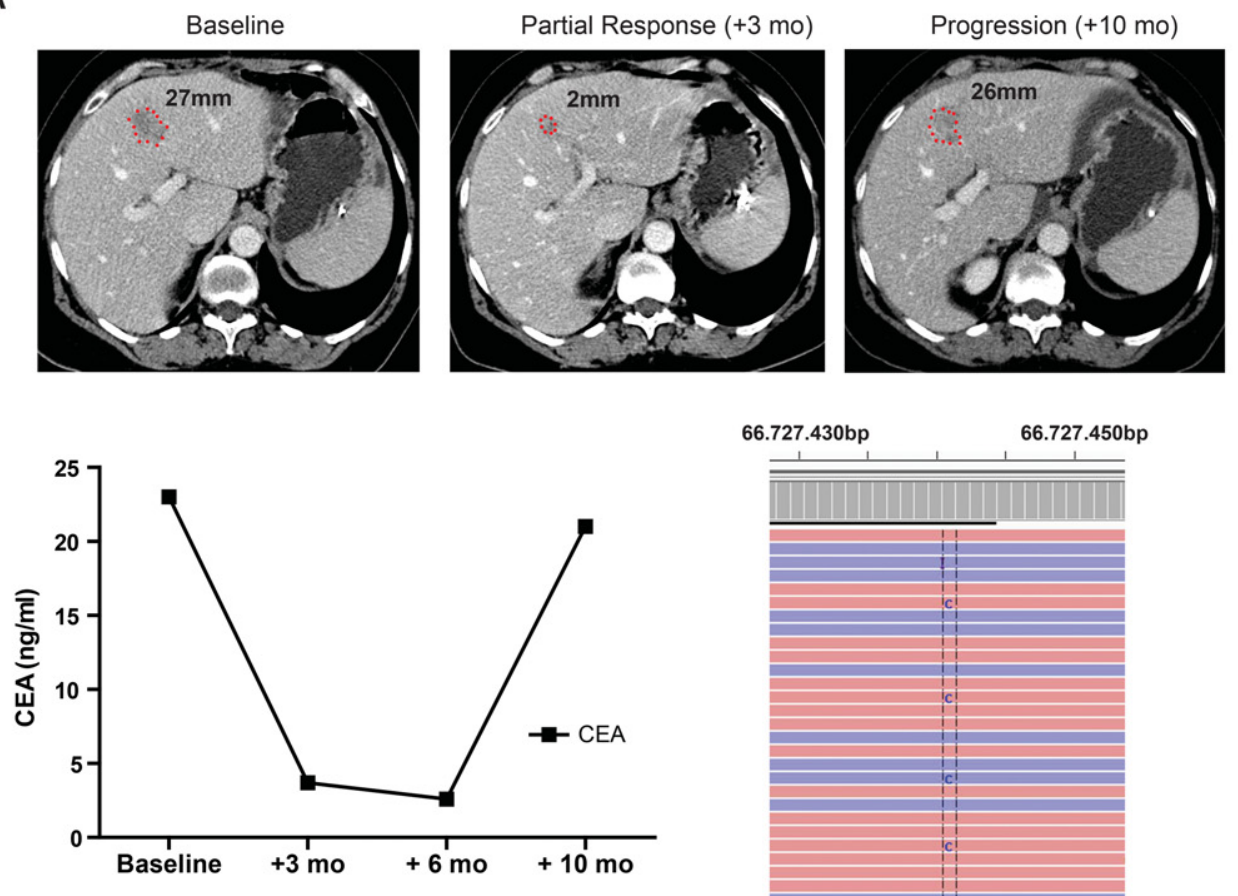

66.727.430bp

$66.727 .450 \mathrm{bp}$

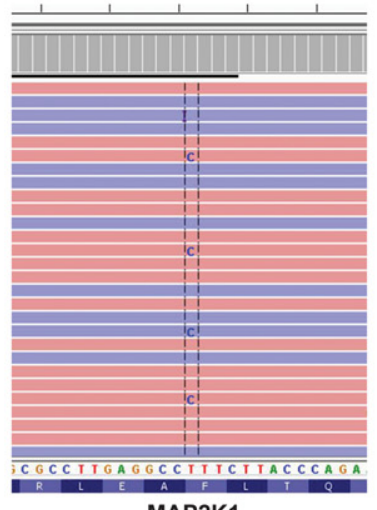

MAP2K1

B
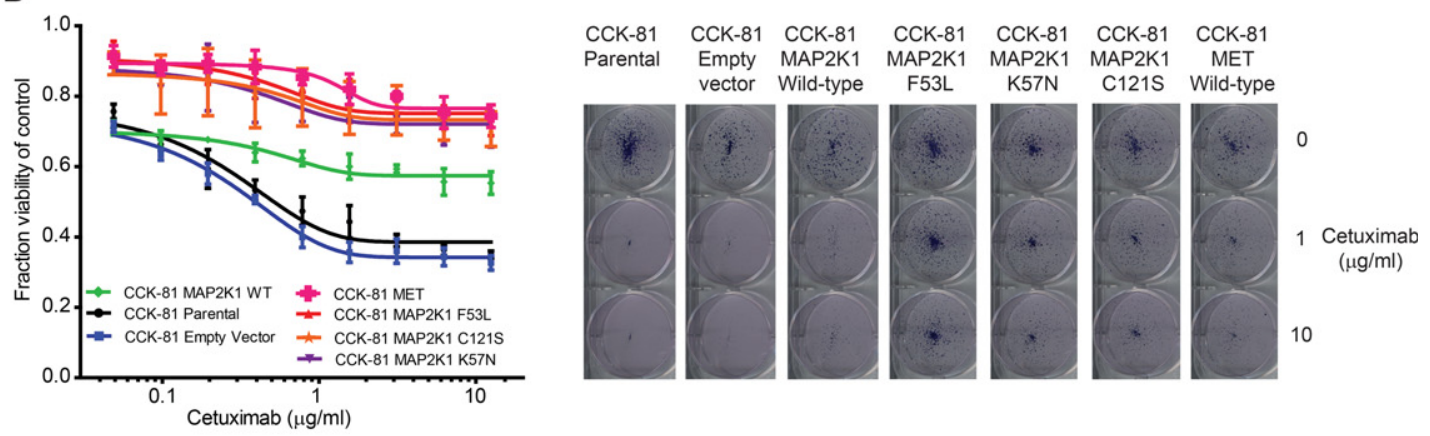

C

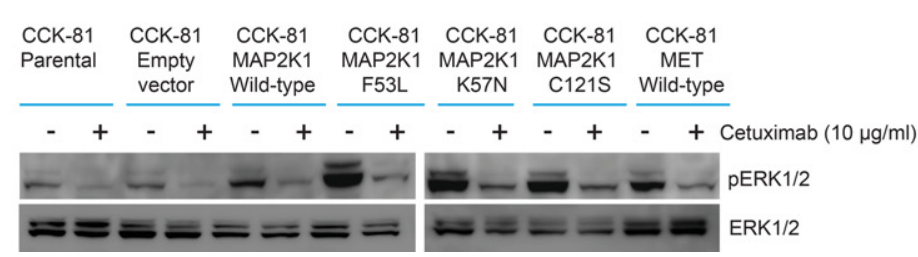

Figure 4. ENU mutagenesis identifies a novel MAP2K1 resistance mutation that is detected in a colorectal cancer patient after an initial response to Cetuximab. (A) A colorectal cancer patient with inoperable liver metastases was treated with the combination of FOLFIRI chemotherapy and Cetuximab. Initial response to treatment was demonstrated radiologically at 3 mo and by falling CEA marker levels. Subsequent measurements at 10 mo confirmed progressive disease, and sequencing of plasma DNA detected a novel mutation in MAP2K1, p.F53L. (B) CCK-81 cells were transduced with the following lentiviral MAP2K1 vectors: empty vector (mock), wild-type (WT), K57N, C121S, and F53L mutations. MET was also overexpressed as a positive control. Cells were seeded in 96-well plates and treated for 1 wk with increasing concentrations of Cetuximab $(0-12.5 \mu \mathrm{g} / \mathrm{mL})($ left) or assayed using a clonogenic survival assay at $20 \mathrm{~d}$ at 1 or $10 \mu \mathrm{g} / \mathrm{mL}$ of Cetuximab (right). (C) Immunoblot of the effect of Cetuximab (10 $\mu \mathrm{g} / \mathrm{mL})$ at $6 \mathrm{~h}$ on pERK in MAP2K1 mutant CCK-81 cells (as in B).

the complete catalog of drug resistance effectors to any drug requires in vitro studies that model resistance in the relevant tissue and genetic background. In the past, such in vitro studies featured cell lines that had undergone serial passage in the presence of the candidate drug in order to force the emergence of resistant clones (Ogino et al. 2007; Turke et al. 2010; Katayama et al. 2011; Eberlein et al. 2015). Although such studies have successfully identified clinically relevant mechanisms of drug resistance in some

\section{Genome Research}

www.genome.org 
A
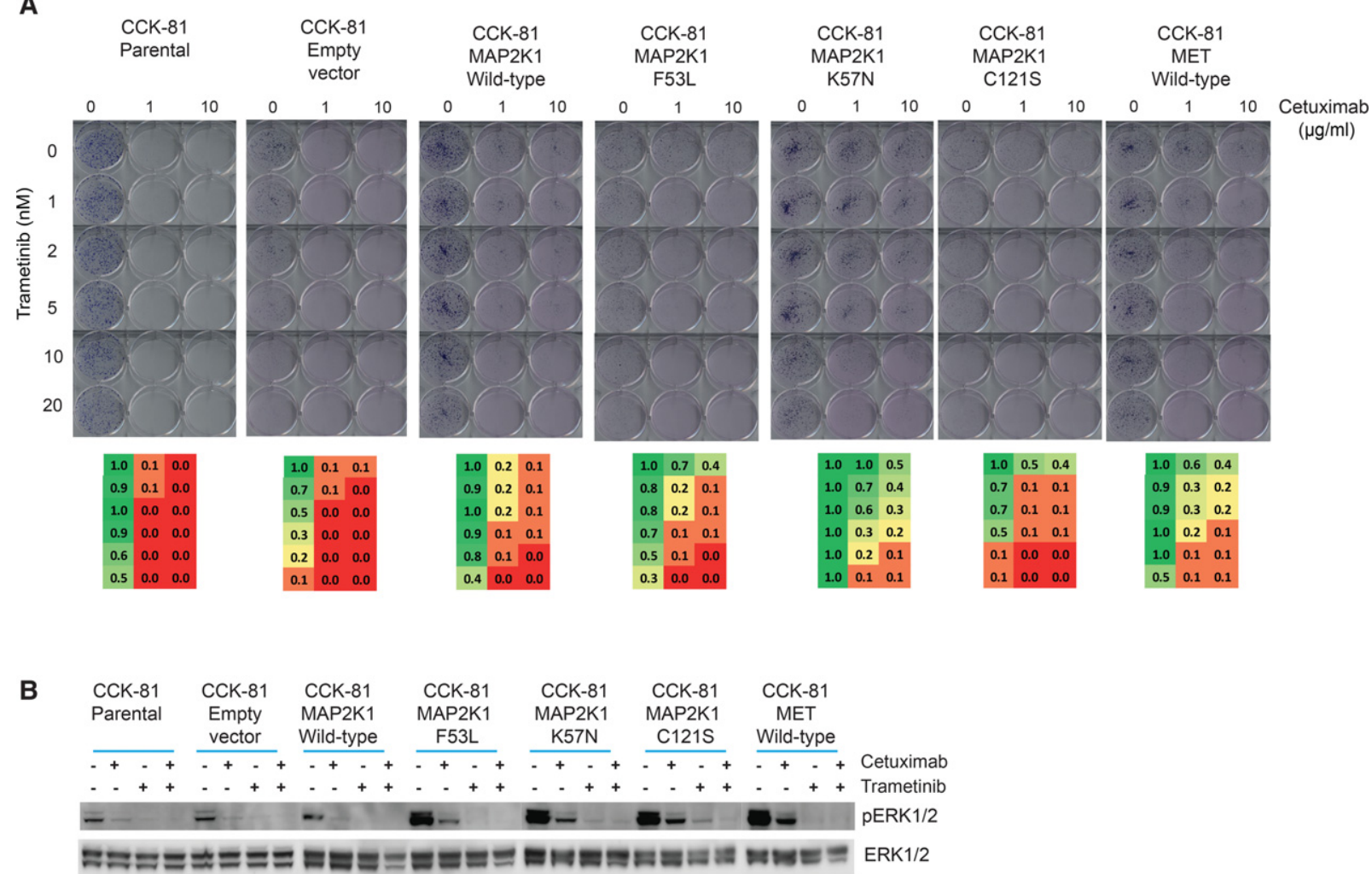

Figure 5. Rational targeting of resistance pathways to resensitize drug-resistant mutants. ( $A$ ) Clonogenic survival assay of $M A P 2 K 1$ mutant CCK-81 cells when treated with Cetuximab, Trametinib, or a combination of the two drugs. Heat maps of normalized viability effects per well are shown below each cell line. (B) Immunoblot of the effect of combing Cetuximab and Trametinib (MEK inhibitor) on MAPK signaling in MAP2K1 mutant CCK-81 cells. Cells were treated with Cetuximab $(10 \mu \mathrm{g} / \mathrm{mL})$, Trametinib $(5 \mathrm{nM})$, or both for $6 \mathrm{~h}$. MET was also overexpressed as a positive control.

instances, they are biased toward selecting for those preexisting resistant subclones that are particular to that specific cell line.

As a means of generating random point mutations throughout the genome, ENU chemical mutagenesis and subsequent phenotype-driven screening has been pivotal to a complete understanding of how complex biological processes operate in classical model organisms including yeast (Forsburg 2001), flies (St Johnston 2002), zebrafish (Patton and Zon 2001), and, perhaps most extensively, mice (Kile and Hilton 2005). The alkylating agent $\mathrm{N}$-ethyl- $\mathrm{N}$-nitrosourea (ENU) can introduce a high rate of point mutations into the genome and has two distinct advantages over previously used mutagens. First, it is very efficient, inducing a point mutation every $1-2 \mathrm{Mb}$ throughout the genome in mouse models ( 100-fold higher than the spontaneous mutation rate and threefold higher than X-irradiation) (Concepcion et al. 2004). Second, unlike irradiation, which induces multilocus deletions, ENU is a point mutagen and affects single loci. ENU functions by the transfer of its ethyl group to oxygen or nitrogen atoms in DNA, resulting in misidentification of these ethylated bases during replication. If the mismatch is not repaired, a base-pair substitution results (Justice 2000). To date, the use of ENU to define drug resistance mechanisms in cancer has been focused on specific genes in noncancer cell line models rather than to interrogate the entire coding genome (Tiedt et al. 2011; Zhang et al. 2011; Ercan et al. 2015). Previous sequencing studies of ENU-derived mutations in mouse and fly models demonstrated a strong bias in the spectrum of substitution mutations observed, with especially low numbers of $C>G, T>G$ and $\mathrm{C}>\mathrm{A}$ mutations. We observed a much more balanced represen- tation of all six possible base substitution contexts in our human cancer cells following ENU exposure (Supplemental Table S8). There should therefore be greater potential to identify a larger number of resistance mutations regardless of their mutation spectrum. Additionally, we compared the mutation spectrum of ENU to that of another mutagen, namely gamma irradiation. Two cancer cell lines (MSI versus MSS) were irradiated, and single cells were expanded to clones prior to exome sequencing. We observed that the mutation spectrum differed quite significantly for the two cell lines, suggesting that unlike ENU mutagenesis, there is a greater cell line-specific effect on the pattern of mutations observed (Supplemental Fig. S10). This would need to be taken into account if gamma irradiation was used for detection of drug resistance genes.

Because it is likely that only one allele is mutated for any specific gene, and $<5 \%$ of the ENU mutations are capable of abrogating protein expression (i.e., nonsense, frameshift, or essential splice site mutations), the screen is strongly biased toward gainof-function or dominant point mutations. Loss-of-function resistance genes would therefore be better captured through genomewide CRISPR inactivation screens or to use ENU in the setting of haploid cell models (although how relevant these are for aneuploidy cancer cells might be a confounding issue).

A major challenge in the interpretation phenotype-directed screening of ENU mutation models is to identify driver mutations from passenger mutations. This is of particular importance in our experiment because ENU mutagenesis generates an average of almost 500 new mutations per drug-resistant clone. We posited 
that in addition to recurrence, evidence that multiple mutations were enriched within the same network or pathway in a mutually exclusive manner would increase the likelihood of these being driver events. There is a long history of using public resources of such networks to identify enriched genes. We used an algorithm (SLAPenrich) to model the likelihood of observing a given number of samples with mutations in the pathway under consideration through a Poisson binomial distribution. It is important to note that identifying resistance mutations in such ENU mutagenesis data is heavily dependent on recurrence of the same mutations across multiple samples; therefore, identification of rare resistance mutations will be challenging unless massive numbers of resistance clones are generated and sequenced. This is especially pertinent with respect to the 30 drug-resistant CCK-1 or NCH-H508 clones, in which SLAPenrich was unable to detect statistical enrichment of mutations in specific pathways. We suggest at least two plausible explanations: (1) These are rare (and therefore not recurrent) mutations and therefore do not cluster into previously characterized pathways interrogated by SLAPenrich; and (2) the observed resistance is the result of mutations outside of the coding exome (for example, in enhancer/promoter or untranslated [UTR] regions) and therefore not amenable to detection using this whole-exome capture approach. With respect to the former possibility, we re-ran SLAPenrich after removing any variants in the previously identified putative resistance genes (BRAF, KRAS, NRAS, MAP2K1, etc.) to increase the power to detect additional enriched pathways; we found nine pathways that were enriched when considering a far less stringent significance threshold (FDR $<20 \%)$. These were dominated by pathways of neurotransmitter signaling (Supplemental Table S9). Given what we know today about EGFR signaling, these are not plausible candidates to confer resistance to Cetuximab. Finally, investigating the possibility of noncoding mutations as resistance drivers would require whole-genome sequencing of these hypermutated samples (each harboring $\sim 50,000$ mutations per genome) and the development of statistical approaches for recurrence detection in noncoding regions. Such algorithms are currently under development as part of the PanCancer Analysis of Whole Genomes (http://pancancer.info/) which is undertaking the analysis of more than 2000 whole genomes. Thus, in due course as whole-genome sequencing costs fall and analytical tools are developed for the noncoding genome, these ENU clones that harbor possible noncoding resistance point mutations could be resequenced and reanalyzed.

Here, we establish a model for the use of genome-wide chemical mutagenesis screens to capture the diversity of clinically relevant drug resistance protein-coding mutations in aneuploid cancer cells. As proof of concept, we used this screen in the setting of an EGFR therapy and colorectal cancer, a disease in which response to such therapy is invariably followed by the acquisition of resistance. Such resistance mechanisms are heavily dominated by point mutations in the MAP kinase signaling pathway and have been extensively validated in patient cohorts (Supplemental Table S1; Yonesaka et al. 2011; Diaz et al. 2012; Misale et al. 2012; Montagut et al. 2012; Bardelli et al. 2013; Bettegowda 2014). We are able to identify all clinically detected resistance mutations to Cetuximab treatment in colorectal cancer, and in addition, potential therapeutic avenues to resensitize resistant cells. We propose that ENU mutagenesis should be incorporated alongside newer genome-wide CRISPR gene editing technologies in the systematic interrogation of drug resistance given the prevalence (and potential for therapeutic targeting) of point mutations as mediators of resistance in cancer.

\section{Methods}

\section{Materials}

All cell culture was performed in either RPMI or DMEM/F12 medium (according to the supplier's recommendations) and supplemented with 5\% FBS and penicillin/streptavidin. Cells were maintained at $37^{\circ} \mathrm{C}$ and $5 \% \mathrm{CO}_{2}$ during culture. The identity of all cell lines used in this paper was confirmed using a panel of 95 single nucleotide polymorphisms (SNPs) used previously for cell line authentication (Fluidigm).

\section{Immunoblotting}

Differential phosphorylation of proteins in signaling pathways were analyzed by Western blot. Cells were plated $24 \mathrm{~h}$ prior to drug treatment and incubated for indicated times and concentrations. Adherent cells were then washed with PBS and collected after indicated incubation time with drug using lysis buffer containing $5 \% \beta$-mercaptoethanol, $150 \mathrm{mM} \mathrm{NaCl}, 50 \mathrm{nM}$ Tris $\mathrm{pH}$ 7.5, $2 \mathrm{nM}$ EDTA pH 8, $25 \mathrm{nM} \mathrm{NaF}, 1 \% \mathrm{NP}-40$, protease inhibitors (Roche), and phosphatase inhibitors (Roche). Lysates were then normalized after bicinchoninic acid (BCA) assay using lysis buffer. Protein lysates were resolved using SDS PAGE electrophoresis in precast Invitrogen $4 \%-12 \%$ Bis-Tris gels and transferred for 12 h. Primary antibodies: p44/42 MAPK, Phospho-p44/42 MAPK (Thr202/Tyr204), and AKT were sourced from Cell Signaling, and phospho-AKT (pS473) was sourced from Invitrogen. Monoclonal beta tubulin was sourced from Sigma.

\section{Drug sensitivity assays}

Cells were seeded in 96-well plates for 6-d assays and six-well plates for 20 -d clonogenic assays. Cells were incubated in drug-free media to allow for adherence for $24 \mathrm{~h}$ before the addition of drug at indicated concentrations. Each cell line was seeded to achieve $\sim 70 \%$ confluency at the end of the assay. Cetuximab was obtained from the Addenbrookes' Hospital Pharmacy. Trametinib (GSK1120212) and Dabrafenib were obtained from Selleckchem.

\section{ENU mutagenesis of cell lines}

The CCK-81 and NCI-H508 cell lines were incubated in a concentration range of ENU $(0-10 \mathrm{mg} / \mathrm{mL})$, and viability was measured after $48 \mathrm{~h}$. A concentration of $0.1 \mathrm{mg} / \mathrm{mL}$ was subsequently selected for resistance models as having a modest effect on cell viability while still generating a high rate of mutations (Supplemental Fig. S11). Cells were incubated in ENU at the indicated concentration for $24 \mathrm{~h}$ before being washed three times with PBS and incubated in media for a further $24 \mathrm{~h}$. Cells were then selected with $10 \mu \mathrm{g} / \mathrm{mL}$ Cetuximab $48 \mathrm{~h}$ post-ENU exposure for $8 \mathrm{wk}$. Clones were then picked using Scienceware small cloning cylinders and either transferred to 96-well plates or expanded into large flasks for drug sensitivity assays. DNA was extracted in 96-well plate format using the Agencourt DNAdvance Genomic DNA Isolation kit.

\section{Gamma radiation of cell lines}

The MSI colon cancer cell line HCT116 and the MSS lung cancer line NCI-H3122 were each irradiated with either 1 Gy or 10 Gy. The following day, single cells were flow sorted and expanded as colonies. DNA was extracted from nine colonies from each cell line and submitted for sequencing.

\section{Genome Research}

www.genome.org 


\section{Whole-exome sequencing}

Exome sequencing was carried out using the Agilent SureSelectXT Human All Exon 50-Mb bait set. Seventy-two clones were DNA extracted and underwent library construction, flow cell preparation, and cluster generation according to the Illumina library preparation protocol. We performed 75-base paired-end Illumina sequencing. Read alignment to the reference human genome (GRCh37) was performed using the Burrows-Wheeler Aligner (BWA) (http://bio-bwa.sourceforge.net/) (Li and Durbin 2010). Unmapped reads were excluded from the analysis. The average coverage across CCK-81- and NCI-508-derived clones was $65 \times$ and $62 \times$, respectively. The matched parental cell lines were sequenced at greater depth (158× in CCK-81 and $144 \times$ in NCI-H508).

\section{Variant detection}

Single-nucleotide substitutions were called using the CaVEMan C (Cancer Variants through Expectations Maximisation) algorithm, and insertions/deletions were called using split-read mapping implemented in the Pindel algorithm (https://github.com/cancerit). The CaVEMan algorithm only analyzes reads that are properly paired and not marked as duplicates. Variants were identified by comparison to a reference single-matched sample consisting of a high sequence coverage contemporary parental cell line control.

\section{Data filtering to remove preexisting subclonal variants}

A number of clones shared mutations which were present in a small percentage of reads in their corresponding contemporary parental cell line sequence. These subclonal mutations could confound subsequent pathway analysis by causing enrichment in a pathway due to mutations that were present before ENU treatment but were not called due to their low representation. To overcome this problem, variants were filtered against the deep sequenced contemporary parental control after mutation calling via CaVEMan and Pindel. The SAMtools mpileup algorithm was used to remove any mutations which were present in $0.5 \%$ or more reads in the high coverage parental cell line control ( $\mathrm{Li}$ et al. 2009). The final set of mutations was used to generate an event matrix for all 72 clones (Supplemental Table S6) and used as the input file for the SLAPenrich analysis described below.

\section{Deciphering mutational signatures branding exome sequences of clones exposed to ENU}

The immediate $5^{\prime}$ and $3^{\prime}$ sequence context of base substitutions identified across Cetuximab-resistant clones was extracted using the Ensembl Core APIs for human genome build GRCh37 and was used to generate mutational catalogs for the downstream analysis. The mutational catalog of CCK-81 Cetuximab-resistant clones contained 7198 substitutions, whereas the NCI-H508 clones contained a total of 23,862 substitutions. Mutational signatures were deciphered separately across both catalogs of mutations, using a previously developed computational framework (Alexandrov et al. 2013b). Briefly, the algorithm identifies a minimal set of mutational signatures that optimally explains the proportions of mutation types found across a given mutational catalog (i.e., across all substitutions identified in CCK-81 and NCI-H508 clones) (Supplemental Fig. S4) and then estimates the contribution of each identified signature to a mutation spectra of each sample included in analysis (i.e., to a mutation spectra of each individual clone; see for NCI-H508 clones) (Supplemental Fig. S5).

\section{Sample level analysis of pathway enrichments (SLAPenrich)}

A short description of the statistical model implemented in SLAPenrich is included in the Supplemental Methods, together with specifications of input and parameter settings used for the analyses presented in this manuscript. SLAPenrich is implemented as an $\mathrm{R}$ package, and it is publicly available at https://github.com/saezlab/SLAPenrich. The computational pipeline to reproduce the presented results is implemented in the enclosed BrammeldEtAl_analysis.R script (also available in the Supplemental Methods).

Briefly, as a first step, SLAPenrich estimates the probability of observing at least one gene belonging to a given pathway mutated in a given sample, based on the length of the total exon blocks of the genes in that pathway, and the sample mutation burden. Once this probability has been estimated for each individual sample, SLAPenrich models the likelihood of observing a given number of samples with mutations in the pathway under consideration through a Poisson binomial distribution. This is the discrete distribution of a sum of Bernoulli trials in which the probability of success is not constant. It is used by SLAPenrich to compute the deviance of the number of observed samples with mutations in a given pathway from its expectation through a corresponding $P$ value assignment.

\section{Identification of drug resistance genes based on the impact of coding mutations}

To identify recurrently mutated driver genes, a $d_{\mathrm{N}} / d_{\mathrm{S}}$ method that considers the mutation spectrum, the sequence of each gene, the impact of coding substitutions (synonymous, missense, nonsense, splice site), and the variation of the mutation rate across genes were used. Owing to the lack of a neutral reference for the indel rate in coding sequences, a different approach was required (for details, see Supplemental Methods). Because this approach has been developed for the detection of driver genes in large cancer sample data sets, samples sharing more than three mutations (i.e., related subclonal populations) were merged to create a single set of variants composed of the union of all observed variants in the similar samples. In the case of the 72 ENU clones, this resulted in the creation of 19 informative samples that were used for the $d_{\mathrm{N}} / d_{\mathrm{S}}$ analysis.

To detect genes under significant selective pressure by either point mutations or indels, for each gene, the $P$-values from the $d_{\mathrm{N}} / d_{\mathrm{S}}$ analysis of substitutions and from the recurrence analysis of indels were combined using Fisher's method (Fisher 1922). Multiple testing correction (Benjamini-Hochberg FDR) was performed separately for all genes, stratifying the FDR correction to increase sensitivity (Benjamini and Hochberg 1995; Sun et al. 2006). To achieve a low false discovery rate, a conservative $Q$-value cutoff of $<0.05$ was used for significance and considered significant any gene with qmis_sfdr $<0.05$ OR qglobal_sfdr $<0.05$. Please see Supplemental Methods for detailed explanations of these methods.

\section{Site-directed mutagenesis of $M A P 2 K 1$ expression vectors}

In order to validate candidate drug resistance mutations from the ENU-based forward genetic screen, we sought to create mutated vectors to express within Cetuximab-sensitive colorectal cell lines. Wild-type construct for MAP2K1 was ordered from Dharmacon and taken forward for in vitro site-directed mutagenesis reactions using the GENEART Site-Directed Mutagenesis System from Thermo Fisher Scientific. To achieve this, two complementary mutagenic oligonucleotide primers were designed (obtained from Sigma-Aldrich) and used to generate gene cDNA expression 
constructs with desired mutations. Mutations were confirmed using Sanger sequencing, before being delivered into cells using lentiviral infection.

\section{Plasma DNA sequencing}

DNA extraction was performed with QIAmp DNA Mini kit (Qiagen). Library preparation was done with the Oncomine Focus Assay (Thermo Fisher Scientific) following the manufacturer's instructions. After bar coding, libraries were equalized to 100 pM. The sequencing template was prepared using the IonPGMSequencing 200 Kit v2 and sequenced in an Ion Select 318 chip using the PGM Sequencing 200 Kit v2 with 500 flows. Hotspot mutations in 35 genes were targeted using the Oncomine Focus Assay (Thermo Fisher) (Supplemental Table S7). Variant Caller v4.0.r73742 was used for variant calling with the Ion Reporter Software. All filtered variants were also analyzed with the Integrative Genomic Viewer (IGV v2.3) software.

\section{Data access}

All raw sequence data from this study have been submitted to the European Genome-Phenome Archive (EGA; http://www.ebi.ac.uk/ ega/), which is hosted at the EBI, under accession numbers EGAS00001001743, EGAS00001001744, and EGAS00001001745. SLAPenrich is implemented as a collection of R scripts and functions and it is publicly available at https://github.com/saezlab/ SLAPenrich. The computational pipeline to reproduce the presented results is implemented in the BrammeldEtAl_analysis.R script (also available in the Supplemental Methods).

\section{Acknowledgments}

U.M. was supported by a Cancer Research UK Clinician Scientist Fellowship. I.M. is supported by a Cancer Research UK Fellowship. F.I. was supported by the European Bioinformatics Institute and Wellcome Trust Sanger Institute. J.S.B., S.P., and J.Y. are supported by a European Research Council Synergy Grant. J.S.B. was supported by a fellowship from the Medical Research Council and the Wellcome Trust.

Author contributions: J.S.B. performed the majority of experiments, analyzed the data, and contributed to writing the manuscript. M.P., L.G.A., I.M., and C.M. analyzed the data. S.B. and M.G. designed and ran the combination screen. C.M., A.D., and B.B. carried out and analyzed the plasma sequencing. F.I. designed the computational methods, analyzed the data, and contributed to writing the manuscript. J.S.R. and M.R.S. supervised the analysis. U.M. conceived the project, analyzed the data, and contributed to writing the manuscript.

\section{References}

Acevedo-Arozena A, Wells S, Potter P, Kelly M, Cox RD, Brown SD. 2008. ENU mutagenesis, a way forward to understand gene function. Annu Rev Genomics Hum Genet 9: 49-69.

Alexandrov LB, Nik-Zainal S, Wedge DC, Aparicio SA, Behjati S, Biankin AV, Bignell GR, Bolli N, Borg A, Borresen-Dale AL, et al. 2013a. Signatures of mutational processes in human cancer. Nature 500: 415-421.

Alexandrov LB, Nik-Zainal S, Wedge DC, Campbell PJ, Stratton MR. 2013b. Deciphering signatures of mutational processes operative in human cancer. Cell Rep 3: 246-259.

Arena S, Bellosillo B, Siravegna G, Martínez A, Cañadas I, Lazzari L, Ferruz N, Russo M, Misale S, González I, et al. 2015. Emergence of multiple EGFR extracellular mutations during cetuximab treatment in colorectal cancer. Clin Cancer Res 21: 2157-2166.

Bardelli A, Corso S, Bertotti A, Hobor S, Valtorta E, Siravegna G, SartoreBianchi A, Scala E, Cassingena A, Zecchin D, et al. 2013.
Amplification of the $M E T$ receptor drives resistance to anti-EGFR therapies in colorectal cancer. Cancer Discov 3: 658-673.

Benjamini Y, Hochberg Y. 1995. Controlling the false discovery rate: a practical and powerful approach to multiple testing. J R Statist Soc B 57: 289-300.

Bettegowda C. 2014. Detection of circulating tumor DNA in early- and latestage human malignancies. Sci Transl Med 6: $224 \mathrm{ra} 24$.

Concepcion D, Seburn KL, Wen G, Frankel WN, Hamilton BA. 2004. Mutation rate and predicted phenotypic target sizes in ethylnitrosourea-treated mice. Genetics 168: 953-959.

Diaz LA Jr, Williams RT, Wu J, Kinde I, Hecht JR, Berlin J, Allen B, Bozic I, Reiter JG, Nowak MA, et al. 2012. The molecular evolution of acquired resistance to targeted EGFR blockade in colorectal cancers. Nature 486: 537-540.

Douillard JY, Oliner KS, Siena S, Tabernero J, Burkes R, Barugel M, Humblet Y, Bodoky G, Cunningham D, Jassem J, et al. 2013. PanitumumabFOLFOX4 treatment and RAS mutations in colorectal cancer. $N$ Engl J Med 369: 1023-1034.

Eberlein CA, Stetson D, Markovets AA, Al-Kadhimi KJ, Lai Z, Fisher PR, Meador CB, Spitzler P, Ichihara E, Ross SJ, et al. 2015. Acquired resistance to the mutant-selective EGFR inhibitor AZD9291 is associated with increased dependence on RAS signaling in preclinical models. Cancer Res 75: 2489-2500.

Ercan D, Choi HG, Yun CH, Capelletti M, Xie T, Eck MJ, Gray NS, Jänne PA. 2015. EGFR mutations and resistance to irreversible pyrimidine-based EGFR inhibitors. Clin Cancer Res 21: 3913-3923.

Fisher RA. 1922. On the interpretation of $\chi^{2}$ from contingency tables, and the calculation of P. IR Statist Soc 85: 87-94.

Forsburg SL. 2001. The art and design of genetic screens: yeast. Nat Rev Genet 2: 659-668.

$\mathrm{Hu} \mathrm{X}$, Zhang Z. 2016. Understanding the genetic mechanisms of cancer drug resistance using genomic approaches. Trends Genet 32: 127-137.

Iorio F, Garcia-Alonso L, Brammeld J, Martincorena I, Wille D, McDermott U, Saez-Rodriguez J. 2016. Population-level characterization of pathway alterations with SLAPenrich dissects heterogeneity of cancer hallmark acquisition. bioRxiv doi: 10.1101/077701.

Justice MJ. 2000. Capitalizing on large-scale mouse mutagenesis screens. Nat Rev Genet 1: 109-115.

Katayama R, Khan TM, Benes C, Lifshits E, Ebi H, Rivera VM, Shakespeare WC, Iafrate AJ, Engelman JA, Shaw AT. 2011. Therapeutic strategies to overcome crizotinib resistance in non-small cell lung cancers harboring the fusion oncogene EML4-ALK. Proc Natl Acad Sci 108: 7535-7540.

Katayama R, Shaw AT, Khan TM, Mino-Kenudson M, Solomon BJ, Halmos B, Jessop NA, Wain JC, Yeo AT, Benes C, et al. 2012. Mechanisms of acquired crizotinib resistance in ALK-rearranged lung cancers. Sci Transl Med 4: 120ra117.

Kile BT, Hilton DJ. 2005. The art and design of genetic screens: mouse. Nat Rev Genet 6: 557-567.

Kobayashi S, Boggon TJ, Dayaram T, Jänne PA, Kocher O, Meyerson M, Johnson BE, Eck MJ, Tenen DG, Halmos B. 2005. EGFR mutation and resistance of non-small-cell lung cancer to gefitinib. N Engl J Med 352: 786-792.

Li H, Durbin R. 2010. Fast and accurate long-read alignment with BurrowsWheeler transform. Bioinformatics 26: 589-595

Li H, Handsaker B, Wysoker A, Fennell T, Ruan J, Homer N, Marth G, Abecasis G, Durbin R; 1000 Genome Project Data Processing Subgroup. 2009. The Sequence Alignment/Map format and SAMtools. Bioinformatics 25: 2078-2079.

Long GV, Fung C, Menzies AM, Pupo GM, Carlino MS, Hyman J, Shahheydari H, Tembe V, Thompson JF, Saw RP, et al. 2014. Increased MAPK reactivation in early resistance to dabrafenib/trametinib combination therapy of BRAF-mutant metastatic melanoma. Nat Commun 5: 5694.

Misale S, Yaeger R, Hobor S, Scala E, Janakiraman M, Liska D, Valtorta E, Schiavo R, Buscarino M, Siravegna G, et al. 2012. Emergence of KRAS mutations and acquired resistance to anti-EGFR therapy in colorectal cancer. Nature 486: $532-536$.

Misale S, Arena S, Lamba S, Siravegna G, Lallo A, Hobor S, Russo M, Buscarino M, Lazzari L, Sartore-Bianchi A, et al. 2014. Blockade of EGFR and MEK intercepts heterogeneous mechanisms of acquired resistance to anti-EGFR therapies in colorectal cancer. Sci Transl Med 6: 224 ra226.

Montagut C, Dalmases A, Bellosillo B, Crespo M, Pairet S, Iglesias M, Salido M, Gallen M, Marsters S, Tsai SP, et al. 2012. Identification of a mutation in the extracellular domain of the Epidermal Growth Factor Receptor conferring cetuximab resistance in colorectal cancer. Nat Med 18: 221-223.

Nik-Zainal S, Davies H, Staaf J, Ramakrishna M, Glodzik D, Zou X, Martincorena I, Alexandrov LB, Martin S, Wedge DC, et al. 2016. Landscape of somatic mutations in 560 breast cancer whole-genome sequences. Nature 534: 47-54.

\section{Genome Research}

www.genome.org 
Ogino A, Kitao H, Hirano S, Uchida A, Ishiai M, Kozuki T, Takigawa N, Takata M, Kiura K, Tanimoto M. 2007. Emergence of epidermal growth factor receptor T790M mutation during chronic exposure to gefitinib in a non-small cell lung cancer cell line. Cancer Res 67: 7807-7814.

Ohashi K, Sequist LV, Arcila ME, Moran T, Chmielecki J, Lin YL, Pan Y, Wang L, de Stanchina E, Shien K, et al. 2012. Lung cancers with acquired resistance to EGFR inhibitors occasionally harbor $B R A F$ gene mutations but lack mutations in KRAS, NRAS, or MEK1. Proc Natl Acad Sci 109: E2127-E2133.

Patton EE, Zon LI. 2001. The art and design of genetic screens: zebrafish. Nat Rev Genet 2: 956-966.

Russo M, Siravegna G, Blaszkowsky LS, Corti G, Crisafulli G, Ahronian LG, Mussolin B, Kwak EL, Buscarino M, Lazzari L, et al. 2015. Tumor heterogeneity and lesion-specific response to targeted therapy in colorectal cancer. Cancer Discov 6: 147-153.

Shostak K, Chariot A. 2015. EGFR and NF-кB: partners in cancer. Trends Mol Med 21: 385-393.

Siravegna G, Mussolin B, Buscarino M, Corti G, Cassingena A, Crisafulli G, Ponzetti A, Cremolini C, Amatu A, Lauricella C, et al. 2015. Clonal evolution and resistance to EGFR blockade in the blood of colorectal cancer patients. Nat Med 21: 795-801.

St Johnston D. 2002. The art and design of genetic screens: Drosophila melanogaster. Nat Rev Genet 3: 176-188.

Sun L, Craiu RV, Paterson AD, Bull SB. 2006. Stratified false discovery control for large-scale hypothesis testing with application to genomewide association studies. Genet Epidemiol 30: 519-530.

Thress KS, Paweletz CP, Felip E, Cho BC, Stetson D, Dougherty B, Lai Z, Markovets A, Vivancos A, Kuang Y, et al. 2015. Acquired EGFR C797S mutation mediates resistance to AZD9291 in non-small cell lung cancer harboring EGFR T790M. Nat Med 21: 560-562.

Tiedt R, Degenkolbe E, Furet P, Appleton BA, Wagner S, Schoepfer J, Buck E, Ruddy DA, Monahan JE, Jones MD, et al. 2011. A drug resistance screen using a selective MET inhibitor reveals a spectrum of mutations that partially overlap with activating mutations found in cancer patients. Cancer Res 71: 5255-5264.
Tokunaga M, Kokubu C, Maeda Y, Sese J, Horie K, Sugimoto N, Kinoshita T, Yusa K, Takeda J. 2014. Simulation and estimation of gene number in a biological pathway using almost complete saturation mutagenesis screening of haploid mouse cells. BMC Genomics 15: 1016.

Turke AB, Zejnullahu K, Wu YL, Song Y, Dias-Santagata D, Lifshits E, Tosch L, Rogers A, Mok T, Sequist L, et al. 2010. Preexistence and clonal selection of MET amplification in EGFR mutant NSCLC. Cancer Cell 17: 77-88.

Van Allen EM, Wagle N, Sucker A, Treacy DJ, Johannessen CM, Goetz EM, Place CS, Taylor-Weiner A, Whittaker S, Kryukov GV, et al. 2014. The genetic landscape of clinical resistance to RAF inhibition in metastatic melanoma. Cancer Discov 4: 94-109.

Van Cutsem E, Köhne CH, Hitre E, Zaluski J, Chang Chien CR, Makhson A D'Haens G, Pintér T, Lim R, Bodoky G, et al. 2009. Cetuximab and chemotherapy as initial treatment for metastatic colorectal cancer. $\mathrm{N} \mathrm{Engl} \mathrm{J}$ Med 360: $1408-1417$.

Wagle N, Van Allen EM, Treacy DJ, Frederick DT, Cooper ZA, Taylor-Weiner A, Rosenberg M, Goetz EM, Sullivan RJ, Farlow DN, et al. 2014. MAP kinase pathway alterations in $B R A F$-mutant melanoma patients with acquired resistance to combined RAF/MEK inhibition. Cancer Discov 4: 61-68.

Yonesaka K, Zejnullahu K, Okamoto I, Satoh T, Cappuzzo F, Souglakos J, Ercan D, Rogers A, Roncalli M, Takeda M, et al. 2011. Activation of ERBB2 signaling causes resistance to the EGFR-directed therapeutic antibody cetuximab. Sci Transl Med 3: 99ra86.

Zhang S, Wang F, Keats J, Zhu X, Ning Y, Wardwell SD, Moran L, Mohemmad QK, Anjum R, Wang Y, et al. 2011. Crizotinib-resistant mutants of EML4-ALK identified through an accelerated mutagenesis screen. Chem Biol Drug Design 78: 999-1005.

Received July 28, 2016; accepted in revised form February 7, 2017. 


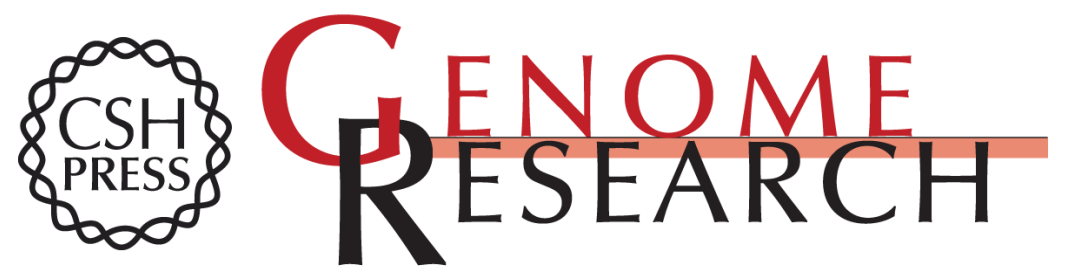

\section{Genome-wide chemical mutagenesis screens allow unbiased saturation of the cancer genome and identification of drug resistance mutations}

Jonathan S. Brammeld, Mia Petljak, Inigo Martincorena, et al.

Genome Res. 2017 27: 613-625 originally published online February 8, 2017

Access the most recent version at doi:10.1101/gr.213546.116

Supplemental
Material http://genome.cshlp.org/content/suppl/2017/03/16/gr.213546.116.DC1

References This article cites 45 articles, 13 of which can be accessed free at:

http://genome.cshlp.org/content/27/4/613.full.html\#ref-list-1

Open Access Freely available online through the Genome Research Open Access option.

Creative This article, published in Genome Research, is available under a Creative

Commons Commons License (Attribution 4.0 International), as described at

License http://creativecommons.org/licenses/by/4.0/.

Email Alerting Receive free email alerts when new articles cite this article - sign up in the box at the Service top right corner of the article or click here.

\section{Affordable, Accurate Sequencing.}

To subscribe to Genome Research go to:

https://genome.cshlp.org/subscriptions 